\title{
A Case Report on Ankyloblepharon Filiforme Adnatum
}

\section{Fateme Eslami $^{1}$, Bahareh Ebrahimi², Pejman Khatibian ${ }^{3}$, Siamak Akbarzade $^{1, *}$}

${ }^{1}$ Assistant Professor, Department of Ophthalmology, School of Medicine, Hamadan University of Medical Sciences, Hamadan, Iran

${ }^{2}$ Resident of Dermatology, Hamadan University of Medical Sciences, Hamadan, Iran

${ }^{3}$ Ophthalmologist, Hamadan University of Medical Sciences, Hamadan, Iran

* Corresponding Author: Siamak Akbarzade, Department of Ophthalmology, School of Medicine, Hamadan University of Medical Sciences, Hamadan, Iran.Email: siakbarzadeh65@yahoo.com

Received: 01.06 .2017 Accepted: 10.09 .2017

\section{How to Cite this Article:} Eslami F, Ebrahimi B, Khatibian P, Akbarzade S. A Case Report on Ankyloblepharon Filiforme Adnatum. Sci J Hamadan Univ Med Sci. 2017;24(3): 259-262. DOI: $10.18869 /$ acadpub.ajcm. 24.3.259.

\section{Abstract}

Background: Ankyloblepharon filiforme adnatum is a rare congenital anomaly characterized by partial or complete adhesion of upper and lower eyelids. This condition is usually observed as an isolated finding; however, it is often associated with other anomalies or a well-defined syndrome. The etiology of this defect has not been identified yet.

Case Presentation: Herein, we reported the case of a four-day-old male neonate who was unable to open his right eye at birth, which was due to adherent upper and lower eyelids as revealed in the physical examination. Family history of the patient was unremarkable. At sterile condition and under anesthesia, the adhesion of the eyelid was cut using a westcott scissor after holding the band of skin with forceps. Systemic and ocular examination did not reveal any abnormalities.

Conclusion: Ankyloblepharon filiforme adnatum is a congenital anomaly that can lead to amblyopia. The accompaniment of this defect with other congenital abnormalities may account for mortality. The early treatment of this anomaly through a simple surgery prevents amblyopia.

Keywords: Ankyloblepharon; Congenital Anomalies 


\title{
تزارش يك مورد انكيلوبلفارون فيليفورم آدناتوم
}

\author{
فاطمه اسلامى'، بهاره ابراهيمى '، يزمان خطيبيان"، سيامك اكبرزادها."

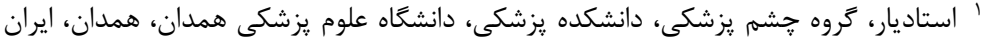

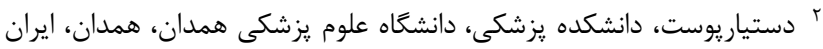

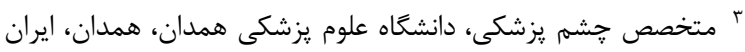

* نويسنده مسئول: سيامك اكبرزاده، گروه جشم يزشكى، دانشكده يزشكى، دانشعاه علوم يزشكى همدان، همدان، ايران. ايميل: siakbarzadeh65@yahoo.com

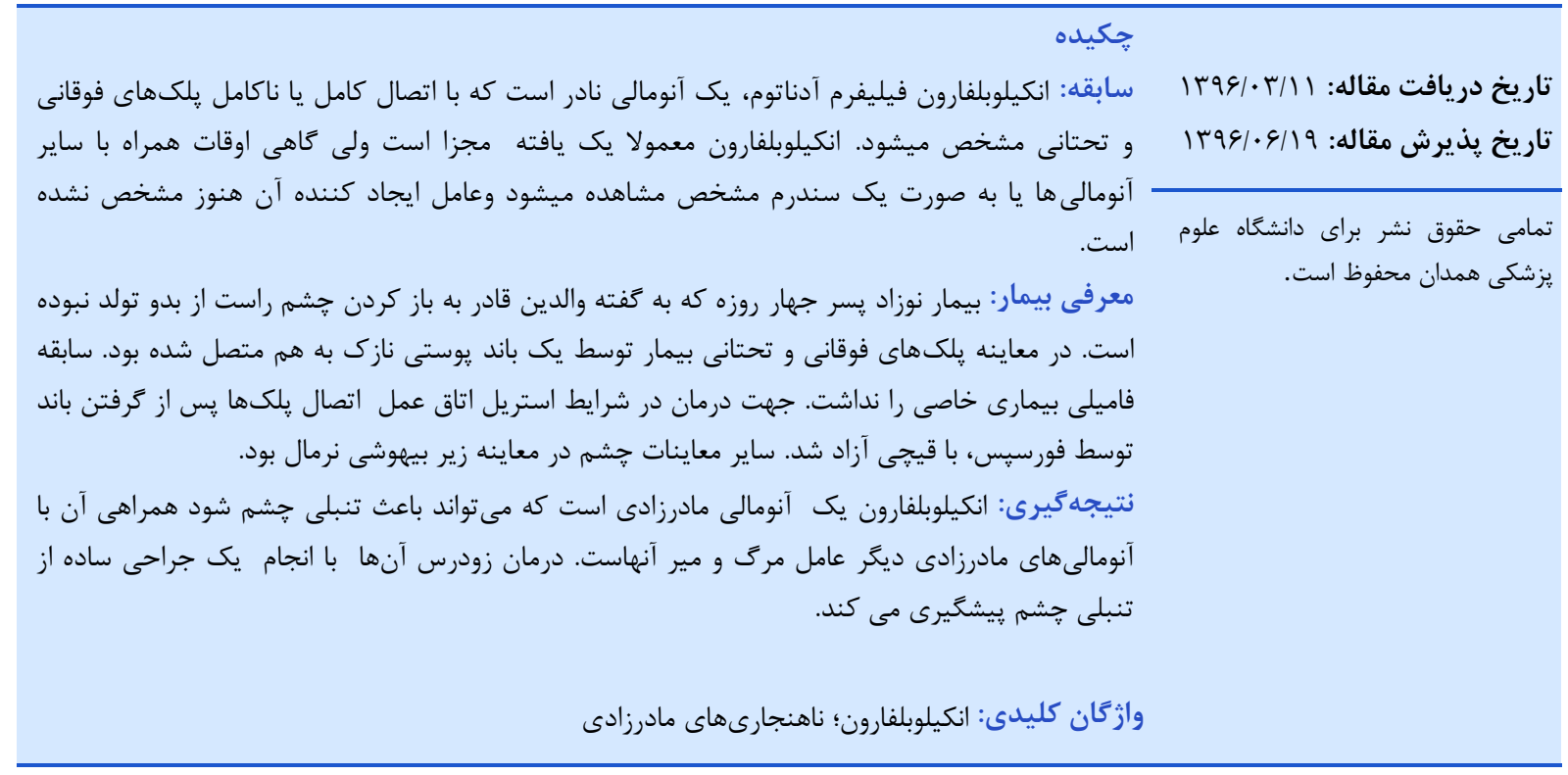

بيمارى AFA بطور موفقيت آميز با جدا كردن يلكها درمان شده است [r-q]

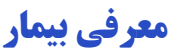

بيمار نوزاد يسر أ روزه كه به كَفته والدين قادر به باز كردن

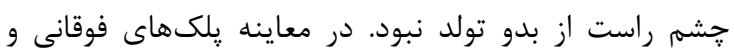

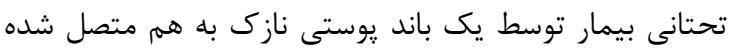

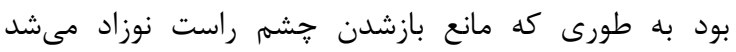

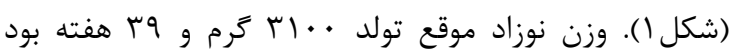

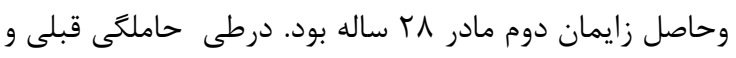

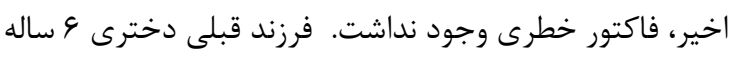

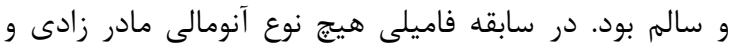

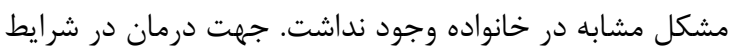

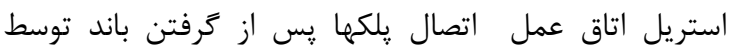

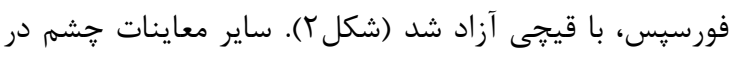

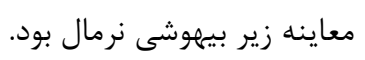

انكيلوبلفارون فيليفرم آدناتوم، يك آنومالى نادر است كه با

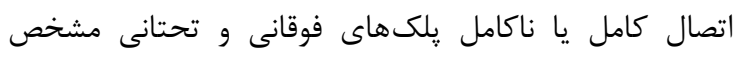

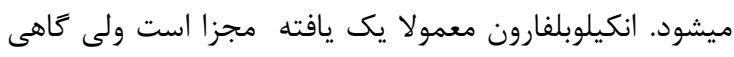

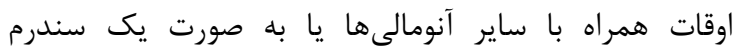

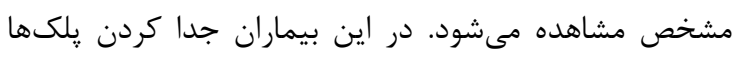

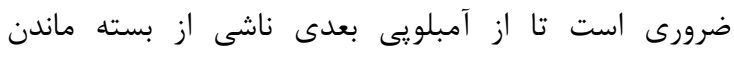
مسير بينايى جلوَيرى شود. انكيلوبلفارون فيليفرم آدناتوم (AFA) Ankyloblepharon filiforme adnatum كامل يا ناكامل لبه سيليارى پرلكهاى فوقانى و تحتانى در ناحيه

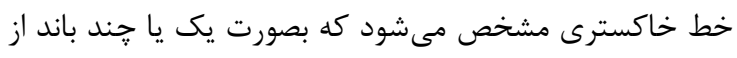

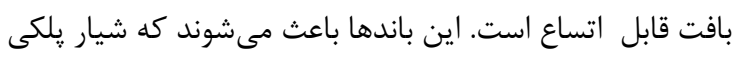

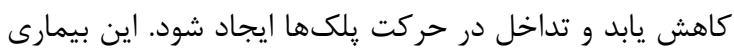

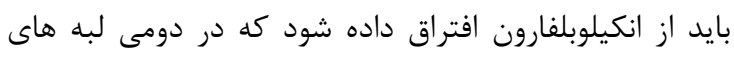

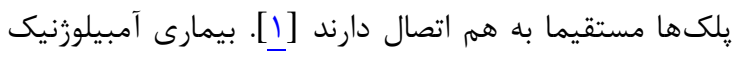

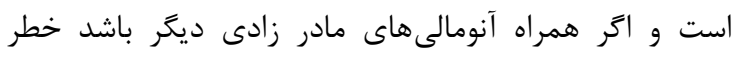

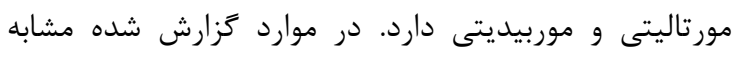




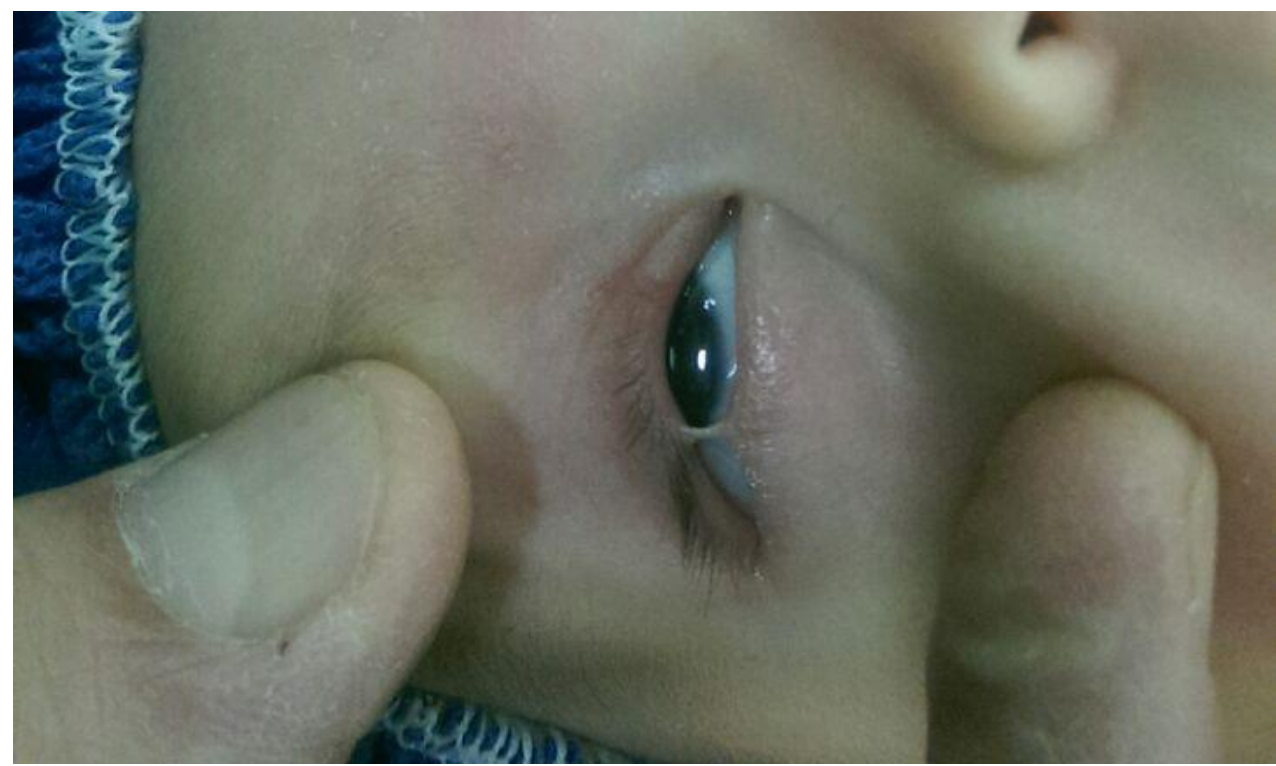

شكل ا: يُلكهاى فوقانى و تحتانى بيمار توسط يك باند يوستى نازك به هم متصل شده است

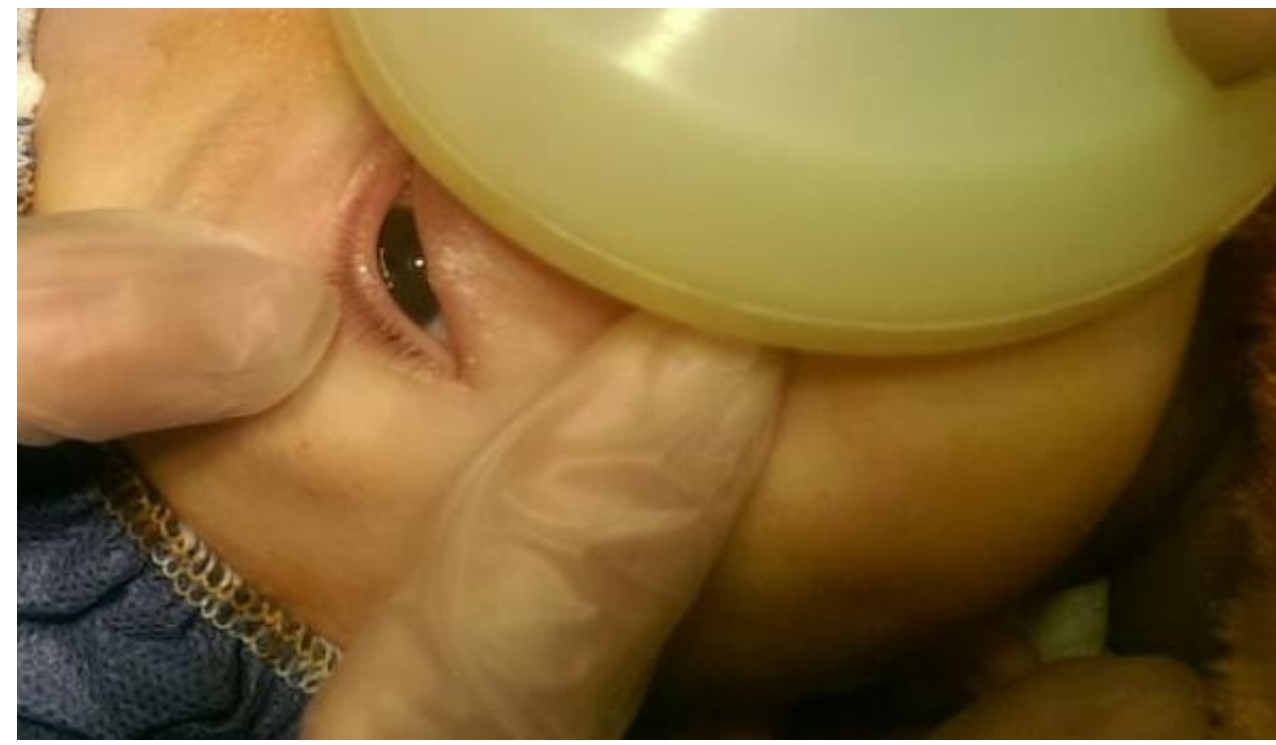

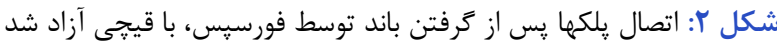

حاشيه اى تركيب شده اند. ولى "كور درو" در بررسى بافت

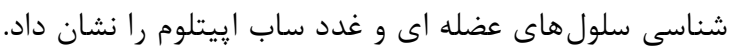

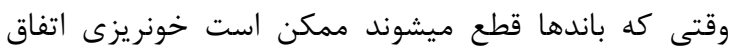
بيفتد [1][ن ياتوزنز دقيق مشخص نشده است. رجندين تئورى مثل

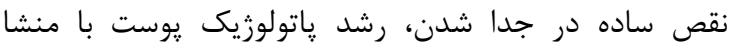

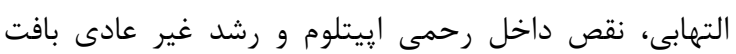

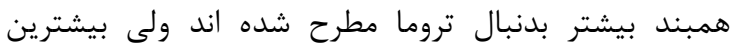

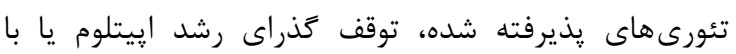

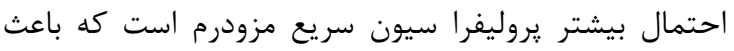
توقف تكامل ميشود [1].] معمولا آنومالى اسيوراديك من منفرد است ولى مئى ميتواند همراه

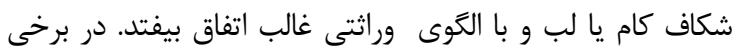

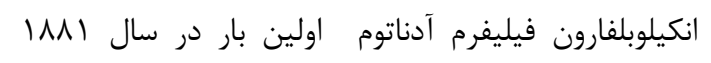

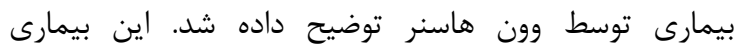
يك آنومالى خوشخيم مادر زادى است كه كه ميتواند باسن

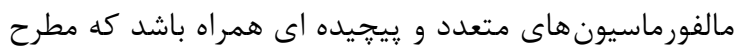

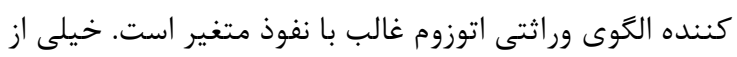
موارد بيمارى بصورت اسيوراديك هستند [ـ[ا]. ارجحيت

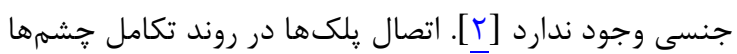
تا ماه ينجم بطور طبيعى اتفاق مى افتد ولى ممكن است جدا

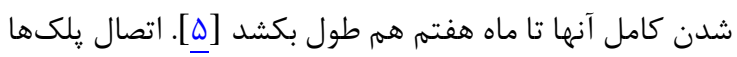

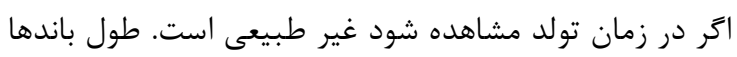

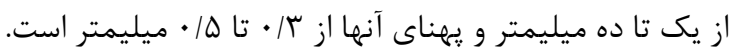
باندها هميشه بين مزه ها و دهانه غدد تارسال كشيده شده

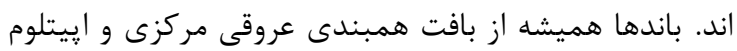


bilateral complete syndactyly of $2^{\text {nd }}$,popliteal pterygia ناخنهاى هييويلاستيك، نييلهاى فرعى و نزول and $3^{\text {rd }}$ toes

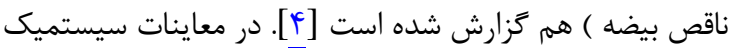

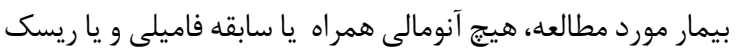
فاكتورى مشاهده نشد.

مشاوره والدين در مورد يترن انتقال زنتيكى بيمارى واحتمال

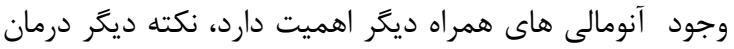

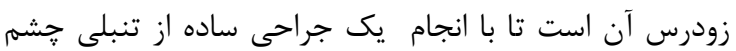
ييشخيرى شود.

\section{REFERENCES}

1. Duke-Elder S. System of ophthalmology. London: Henry Kimpton; 1964. P. 869.

2. Gupta SP, Saxena RC. Ankyloblepharon filiforme adnatum. $J$ All India Ophthalmol Soc. 1962;10:19-21. PMID: 13903005

3. Akkermans $\mathrm{CH}$, Stern LM. Ankyloblepharon filiforme adnatum. Br J Ophthalmol. 1979;63(2):129-31. PMID: 218608

4. Modi AJ, Adrianwalla SD. A multiple malformation syndrome with ankyloblepharon filiforme adnatum, with cleft lip and palate. Indian J Ophthalmol. 1985;33(2):129-31. PMID: 3009323

5. Sharkey D, Marlow N, Strokes J. Ankyloblepharon filiforme adnatum. J Pediatr. 2008;152(4):594. PMID: 18346523 DOI: $\underline{10.1016 / j . j p e d s .2007 .12 .051}$
بيماران جزئى از سندرم (Trisomy 18) ectodactyly- Hay-Wells syndrome ،(ectodermal dysplasia-cleft lip-palate syndrome curly hair- ‘popliteal pterygium syndrome (CHANDS) ankyloblepharon-nail dysplasia مواردى، همراهى با هيدروسفالى، مننگوسل، آنوس ايميرفوره، سين

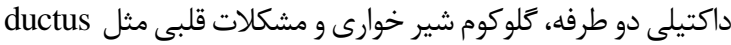
ventricular septal defect garteriosus يك مورد نامعمول جندين سندرم همراه AFA ( شكاف لب و كام،

6. Gruener AM, Mehat MS. A newborn with ankyloblepharon filiforme adnatum: a case report. Cases J. 2009;2:8146. PMID: 20175880 DOI: 10.1186/1757-1626-0002-0000008146

7. Williams MA, White ST, McGinnity G. Ankyloblepharon filiforme adnatum. Arch Dis Child. 2007;92(1):73-4. PMID: 17185446 DOI: $10.1136 /$ adc. 2006.103069

8. Ioannides A, Georgakarakos ND. Management of ankyloblepharon filiforme adnatum. Eye (Lond). 2011; 25(6):823. PMID: 21350566 DOI: 10.1038/eye.2011.26

9. Jain S, Atkinson AJ, Hopkisson B. Ankyloblepharon filiforme adnatum. Br J Ophthalmol. 1997;81(8):705. PMID: 9349166 